\title{
ASSESSMENT METRICS, CHALLENGES AND STRATEGIES FOR MOBILE HEALTH APPS
}

\author{
Chi Zhang, Southern Polytechnic State University, chizhang@spsu.edu \\ Xihui Zhang, University of North Alabama,xzhang6@una.edu \\ Richard Halstead-Nussloch, Southern Polytechnic State University, rhalstea@spsu.edu
}

\begin{abstract}
This paper intends to further our understanding of the current challenges of mobile health applications (mHealth apps) through a systematic review of the existing literature. Starting with an overview of the current challenges of and regulations for health apps, it proposes usability metrics for health app assessment and strategies to address the health app challenges from the points of view of both healthcare consumers and providers. The recommended list of extended metrics and a new classification scheme for the challenges and strategies proposed here can be used as guidance for future research on mobile health app development as well as providing beneficial information for anyone interested in the implementation and assessment of mHealth.
\end{abstract}

Keywords: Usability, Health App, Medical App, Assessment, Mobile Device

\section{INTRODUCTION}

Mobile health is defined by the Global Observatory for eHealth of the World Health Organization as the "medical and public health practice supported by mobile devices including phones, patient monitoring devices, personal digital assistants (PDAs), and other wireless devices" [36]. Mobile health applications (mHealth apps) have become ubiquitous and tens of thousands of health apps are now available for download to mobile devices (e.g., smartphones and tablets); they are used by healthcare professionals, consumers, and patients. Mobile health apps can help people manage their own health, promote healthy living, and gain access to personalized medical information, anywhere and anytime. Mobile health apps are poised to cause a disruptive shift in patient engagement and healthcare delivery [31].

With the Affordable Care Act in effect, a series of rules are scheduled for implementation in 2014. One likely effect of these rules is that mobile health apps will likely play an increasingly significant role in providing convenient access to health information. In the best case, they will help in overcoming communication barriers among healthcare providers, facilities, and patients. This is important for improving care as it is predicted that, by the end of 2014, 9 in 10 healthcare providers will use smartphones and/or tablets in their clinical practice [9]. Clearly, mobile devices will become, if not already, a platform of top choice for provider communications, information, and delivery of healthcare.

On the patient side, according to Pew Research Center's findings, $31 \%$ of cell phone owners look up health information on their smartphones, while $20 \%$ smartphone owners have a health app installed on their smartphones [11]. The report by research2guidance [27] estimates that over 500 million of a total 1.4 billion smartphone users worldwide will be using at least one healthcare app by 2015. By 2018, 50\% of the more than 3.4 billion smartphone and tablet users will have downloaded mobile health apps. Although the patient side has not achieved the $90 \%$ level as observed on the provider side, mobile devices are growing rapidly as a tool for patients in effective healthcare.

As more people own mobile devices and more third-party apps become available, some concerns have been raised. A major concern is that both medical professionals and the general public are overloaded with what is already an abundance of these health apps. As a result, health app users feel that there is, in the terms of a physician we have recently interviewed, a "bewildering array" of health apps available and that furthermore little is currently understood about the huge range and variety of health apps and their role in effective healthcare. The issues are wide-ranging and include, e.g., location and selection of appropriate apps, privacy and security issues, the lack of evaluation standards of the apps, little guidance on their quality, and the pressure for them to move into the mainstream of healthcare and many more [5][18][30][31][32]. 


\section{Issues in Information Systems \\ Volume 15, Issue II, pp. 59-66, 2014}

This paper aims to provide an overview of the current state of mobile health apps based upon recent research. The review focuses on the categorization of the app's functionality, the challenges health apps are facing, the regulations and initiatives on assessment of apps, and the exemplar curation platforms for app review and evaluation. Based on the recent published research, a new classification approach to address mHealth challenges and strategies and a recommended list of usability metrics for health apps are proposed. It is our hope that the compilation and organization of this current information on healthcare apps will improve our understanding of the usefulness and usability of mobile health apps and provide guidance to both their design and implementation.

\section{MOBILE HEALTH APPS CATEGORIZATION AND CHALLENGES}

Because they influence health behaviors in real-time [1], mHealth apps enable leap-frog-size opportunities to improve access to health promotion and interventions. Health apps serve many different purposes, such as providing medical information through a mobile device (e.g., Physician's Desk Reference, WebMD), mobile wellness apps, and the apps designed to access electronic health records (EHR) and personal health records (PHR). Mosa et al. [25] reviewed 83 healthcare apps for smartphones which are documented on MedLine. They categorized the users and purposes of the health apps into three major types: (1) apps for healthcare professionals on disease diagnosis, drug reference, medical calculators, literature search, clinical communication, hospital information system client applications, medical training, and general healthcare; (2) apps for medical or nursing students on medical education; and (3) apps for patients focusing on disease management with chronic illness and other conditions.

All users of the above three types are reported to have usability difficulties, e.g., in keeping an overview of all the medical apps available, finding the right apps for them, and pinpointing the added value in these medical apps [8][12]. App consumers are overwhelmed by the great number of health apps available for download. Lots of them choose not to download any of them because they cannot decide which apps will meet their needs and they do not want to have too many health apps on their mobile devices [32]. So the initial and potentially the greatest challenge for health apps is selection from the existing large inventory of those apps and then integrating those selected into the user's mobile device and its associated use routines.

\section{Healthcare Consumers' Viewpoint}

From the patients' point of view, mobile health apps can help them schedule appointments, access their medical health records or lab tests, drug references, and track health activity and medical news. A recent report on the current situation of health apps shared similar concerns about how healthcare apps can help in the prevention and treatment of disease and thus improve health outcomes. The IMS Institute for Healthcare Informatics released a report in October 2013, "Patient Apps for Improved Healthcare: From Novelty to Mainstream," in which they analyzed more than 40,000 healthcare apps available for download. Of the 40,000+ apps, only 16,275 are directly related to patient health and treatment. The rest are providing data that neither healthcare professionals nor consumers can use to improve their wellbeing [18][30].

The IMS Health report [18] on healthcare apps shows that most apps for patients and general public available to date have been in the overall wellness category pertaining to diet and exercise. The individual functionalities of healthcare apps include to inform (provide information in a variety of media), instruct (provide instructions to the user), record (capture user entered data), display (graphically display user entered data/output user entered data), guide (provide guidance based on user entered information, e.g., recommend a physician consultation or course of treatment), remind/alert (provide reminders to the user), and communicate (provide communication with healthcare provider/patients and/or provider links to social network).

The healthcare apps reviewed by IMS Health also demonstrated the functionality across the patient journey, from prevention to compliance. They include prevention/healthy lifestyle (healthy living, diet and exercise, addiction quitting, stress, relaxation, and sleep), finding a healthcare professional (doctor reviews and referrals, second opinions), diagnosis/education (medical information, condition management information, emotional support postdiagnosis), self-diagnosis (symptom checker and self-diagnosis), filling prescriptions (finding a pharmacy, availability, price and insurance coverage), and compliance (persistence, compliance). 


\section{Issues in Information Systems \\ Volume 15, Issue II, pp. 59-66, 2014}

"The vast majority of available apps has limited functionality or evidence of value in advancing healthcare provision and outcomes" [4, para. 1]. It is widely reported that most health apps are simple in design and do little more than provide information without interactivity between provider and patient, which prevents their broad and systematic use by healthcare providers and patients. It is notable that while some apps provide multi-functionality, less than half of the apps provide even minimal information and instructions. Approximately $20 \%$ of the apps provide information and track or capture user data [4]. It appears that only about 1 in 5 health app is currently positioned to support a meaningful interaction between provider and patient. With such a low baseline rate, there is little wonder that patients and providers are bewildered by the array of health app possibilities. Added to the low baseline rate, an app's utility will range far and wide - from weight loss apps to X-ray apps, to medical information apps, and to personal health apps. With more than hundreds of thousands of health apps to sift, it is sometimes difficult to know where to begin or how to separate the all-stars from the failures. Thus, the primary challenge that patients seem to face is finding mHealth apps that provide positive value for their healthcare outcomes.

\section{Healthcare Providers' Viewpoint}

Healthcare providers have been deploying mobile devices in practice successfully for a number of years [35]. As new payment programs come into effect, providers and payers will need more apps to capture data for payment, managed care and the like. The two top reasons for providers to adopt mobile health technologies are for time efficiency and for cost efficiency. Improving quality and continuity of care, improving communication with patients, and fulfilling patients' demand are placed lower on the list. Many providers, however, are still resisting mobile health because they are not yet convinced that the technology can help them become successful or improve on their current success. Still, it is remarkable to see how many tablets and mobile devices are being used in healthcare today [17].

According to a new report by MedData Group, a healthcare marketing firm, about two-thirds of the 532 surveyed physicians use some kind of mobile applications in the performance of their jobs and almost $60 \%$ physicians are considering using mobile access to EHR in the next 12 months [17]. These findings provide a snapshot of which mobile technologies physicians are using and what they want to use in the near future. For physicians of all specialties, the most often used mobile healthcare application is for medication interactions, with these apps being used by nearly $50 \%$ of the doctors surveyed. The leading use of the technology appears to help doctors make prescription decisions and be diligent in doing so. The other frequently used types of applications are for diagnosis, to access EHR, labs/images/tests, clinical notes, and ePrescribing. The MedData Group also found what doctors want to use in the near future: mobile access to EHR, apps for secure texting, point-of-care information on drugs, devices, or diagnoses, and patient portals. The doctors also want to have apps for mobile health monitoring and patient eVisits via mobile devices.

To summarize, the use of health apps by healthcare professionals and the general public has both benefits and challenges. Besides the benefits, the major challenges associated with health apps include:

1. Selecting health apps that provide value from the wide range of possibilities [18]

2. Health apps designed without consideration of users with low health literacy [2]

3. Information provided by health apps may not be accurate and accountable [26]

4. Privacy and security concerns [7][20]

5. Health apps lack evaluation [18]

6. Download and use of the health apps without any guidance [16]

7. Usability constraints such as small screens, difficulty in reading and typing, slow download speed [22]

\section{HEALTH APP DEVELOPMENT STRATEGIES AND USABILITY EVALUATION}

In order to meet the challenges and solve the problems, regulators and policymakers have been working on the initiatives, strategies, and guidelines on health app development. The FDA released the "Mobile Medical Applications: Guidance for Industry and Food and Drug Administration Staff" on September 25, 2013. The FDA's focus is on the apps that present greater risk to patients if they do not work as intended and on apps that cause smartphones or other mobile devices to impact the functionality or performance of traditional medical devices [10]. 
It is believed that at least four areas must be addressed as mHealth development strategies [18]:

1. Payers and providers, as well as regulators and policymakers, need to recognize the role apps can play in healthcare.

2. Security and privacy guidelines and assurances must be established among providers, patients, and app developers.

3. There needs to be a systematic evaluation of apps to inform users of their appropriate use.

4. Apps must be effectively integrated with other aspects of patient care.

Strategy \#3 listed above is closely related to evaluation and selection of health apps. The mHIMSS App Usability Group published a guide in July 2012 on the usability of apps. The selection of apps may ultimately be evaluated based on a number of factors including price, user reviews, usability, and security [24].

Usability is one of the four factors for evaluating health apps as specified by mHIMSS; usability is defined as "the effectiveness, efficiency, and satisfaction with which specific users can achieve a specific set of tasks in a particular environment" [24, p. 5]. Over the past thirty-five years, different standards or models have been proposed to quantify and assess usability. Examples include ISO 9241-11 [19] standard, which takes a broader perspective on usability measurement and defines usability as whether users can achieve specified goals with efficiency, effectiveness, and satisfaction in a specified context of use. Effectiveness refers to the accuracy and completeness with which users achieve specified goals. Efficiency measures the resources expended in relation to the accuracy and completeness with which users achieve goals. Satisfaction assesses freedom from discomfort, and positive attitudes towards the use of the product [15].

Within healthcare, the measurable objectives of health app usability should be considered along with the healthcare practice goals. In the context of healthcare applications, the following example questions could be asked to measure the health app usability [24]:

1. How many steps does it take to use the app for prescription refill requests? (efficiency)

2. How well the patient-encounter data are used for patient education? (effectiveness)

3. Would you use the data obtained from the app in your clinical decision making process? (user satisfaction)

4. How much training will be required for clinicians to become adept with the app? (ease of learning)

Furthermore, mHIMSS [24] specifies nine usability principles in terms of design of mobile applications: simplicity, naturalness, consistency, forgiveness and feedback, effective use of language, efficient interactions, effective information presentation, preservation of context, and minimized cognitive overload. mHIMSS also suggests that typical clinical scenarios can help assess an application's usability.

In addition to the consideration of practice goals and measurable objectives and assessment of the app's usability with typical clinical scenarios, mHIMSS's guide to evaluating app usability advises checking other resources such as an app-curation platform to supplement app market reviews. There are some publicly available medical app review platforms that gather reviews and ratings based on user satisfaction data. Digital content curation is a process of analyzing and sorting Web content and presenting it in a meaningful and organized way around a specific theme [21]. Similarly, a mobile app curation helps solve the problem of fragmented, time consuming, and laborious process of finding an appropriate app [3].

The following is a list of a few trustworthy resources for health app review [24]:

- iMedicalApps - reviews with indexed search functions by app type, physician specialty, and device platform; reviewed and generated by physicians

- Medical Apps on iTunes - reviews focused on iPhone medical apps

- Happtique - a mobile medical app store developed by healthcare professionals intending to help both healthcare providers and health app developers

- MobiHealthNews - news and reviews on the latest medical apps

- Aetna's CarePass - open to the public and integrates a number of health apps with shared data

The guide proposed by mHIMSS is the first step towards standardizing usability measurement and metrics for mobile health apps. It is necessary and critical to have a framework for assessing the usability of medical apps through formal and scientific means. The NIST Health IT Usability initiative is focused on establishing a framework 


\section{Issues in Information Systems \\ Volume 15, Issue II, pp. 59-66, 2014}

that defines and assesses health IT usability. The initiative examines the human factors that are critical to designing usable EHRs and will guide industry in usability engineering practices. The NIST has published the usability guide "Technical Evaluation, Testing, and Validation of the Usability of Electronic Health Records" in 2012 [23]. It is expected that the NIST will issue another evaluation guide specifically on mobile health apps in the near future.

\section{A PROPOSED CLASSIFICATION SCHEME}

To aid solving the problems of current health apps summarized above, we propose a classification scheme (Table 1) incorporating our findings on the challenges of mobile health apps and strategies to ensure appropriate design and development of the apps for healthcare providers, patients, and the general public. The classification scheme can be used as a guideline for future research on mobile health app development and also as guidance for mHealth app implementation.

Table 1. Classification of Health App Challenges and Solution Strategies

\begin{tabular}{|c|c|c|}
\hline $\begin{array}{l}\text { Category of } \\
\text { Challenges }\end{array}$ & Challenges & Solution Strategies \\
\hline App overload & $\begin{array}{l}\text { Difficulty in keeping an } \\
\text { overview of all the medical apps } \\
\text { available } \\
\text { Difficulty with finding the right } \\
\text { app and integrating it with the } \\
\text { user's current mobile device } \\
\text { workflows }\end{array}$ & $\begin{array}{l}\text { - Guidance through formal and scientific } \\
\text { means } \\
\text { - Trustworthy curation platform } \\
\text { Information sharing and peer support } \\
\text { through web-connected virtual } \\
\text { community }\end{array}$ \\
\hline Content & 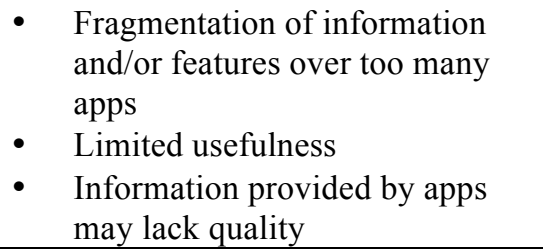 & $\begin{array}{l}\text { - Apps can work as gateway to medical } \\
\text { information by incorporating information } \\
\text { from different sources } \\
\text { - } \quad \text { Standardize medical content } \\
\text { - } \\
\text { Increased regulation to improve } \\
\text { accountability of app content }\end{array}$ \\
\hline $\begin{array}{l}\text { Usability } \\
\text { constraints }\end{array}$ & $\begin{array}{l}\text { Reading from a small screen, } \\
\text { difficult input, and slow interaction } \\
\text { speed }\end{array}$ & $\begin{array}{ll} & \text { User-interface design guidance } \\
\text { - } & \text { Best practices } \\
\text { - } & \text { Usability testing and heuristic reviews } \\
\end{array}$ \\
\hline $\begin{array}{l}\text { Privacy and } \\
\text { security }\end{array}$ & $\begin{array}{l}\text { Privacy, confidentiality, and security } \\
\text { issues }\end{array}$ & $\begin{array}{l}\text { - Regulations, guidance and policy } \\
\text { frameworks } \\
\text { - Uniform privacy and security protection }\end{array}$ \\
\hline
\end{tabular}

Mobile health apps can help consumers, patients, and healthcare professionals in a variety of ways. Health apps assist people manage their own health, promote healthy living, improve access to care and quality of care, and improve staff productivity and sustainability. It is important to have and use a set of systematic evaluation guidelines and a clear vision for health apps to ensure the quality of the apps to all users and especially the consumers and patients. Likewise increasing the trustable recommendations and support from providers and other healthcare professionals for health apps is important. To facilitate these improvements, we propose additional metrics for mobile health app assessment.

\section{PROPOSED ADDITIONAL METRICS FOR HEALTH APP ASSESSMENT}

Usability is believed by the authors to have changed drastically in the mobile environment. The change has been disruptive in the sense that mobile users are now often the sole arbiters of good usability including user experiences. Furthermore, the methodology for human-computer interaction (HCI) and user experience design is ever increasingly diffuse in the mobile environment [14]. The diversity of the specified context of use and specified goals to achieve positive outcomes associated with mHealth apps also requires increased consideration and weight in the usability of mobile environments.

The examples of use attributes and metrics identified by various, existing usability standards and models include those focusing on characteristics of users and tasks and also attributes and characteristics of the technical, physical, 
organizational, and social environments of the human-computer interaction. It has been pointed out that not all of the attributes are relevant for all types of usability evaluation and furthermore, and it is necessary to consider additional attributes for a given type of interactive system such as for mobile applications [29].

Based on the discussion of the current challenges that mobile health apps are facing, the existing usability measurements and metrics for mobile health apps, and the changes of usability in the mobile environment, we propose the following additional metrics for mobile health app assessment:

1. Usefulness. Usefulness enables a user to achieve his or her specific goals and motivates the user to use it again. It is still relevant in mobile environments and the first and foremost success factor for mobile health apps. In the mobile app market, if an app is easy and satisfying to use but does not achieve the goals of a specific user, it will not be used or may be deleted from his or her mobile devices all together even if the app is free.

2. Effectiveness. Effectiveness refers to the extent to which the app behaves in the way that users expect it to and the ease of which users can use it to achieve their specific goals [28]. It might also be a traditional metric but it is important for health apps.

3. Veracity. Veracity refers to accuracy and reliability of the content, data or information to the degree specified in the app. Content in health apps is usually based on one or more information sources. The apps need to provide "a method or citation to enable the user to locate to the complete content" [33]. An app that performs user or patient management functions, such as calculation, data tracking, reminders, or other functions, needs to be consistently accurate and reliable.

4. Interactivity. Interactivity has been examined for website interactivity in improving website usability (e.g., [13]). In the context of mobile health apps, interactivity provides sense of engagement, entertainment, satisfaction, and motivation for the users to use the apps again. It also extends to interactivity between providers and patients as facilitated by the mHealth app.

5. Customization/Personalization. The purpose of a specific health app may be designed to support users in one or more healthcare delivery domain. Examples include assessment, diagnostics, prevention, intervention, and recovery, to name a few. Customization or personalization is important to help the apps achieve what the users intend to do. For example, the apps may need to connect to one or more electronic health records (EHR) systems to provide the medical data or customized subsets of data of a particular patient.

6. User Acceptability or User Satisfaction. User acceptability can be defined as the demonstrable willingness for specified users to use a specific app for the tasks it is designed to support [6]. User acceptability seems to have replaced most of the traditional usability metrics in mobile environment. Traditionally, technology could rely on authority to ensure that technology was used. As discussed earlier, mobile users are now often the sole arbiters of good usability including user experiences.

To prevent or eliminate design problems and minimize potential frustration for users, the proposed additional metrics for mobile app evaluation can be used as a guide for app developers, curation platforms and regulatory agencies. The context of use descriptions for a specified health app should be established iteratively at the early stages to develop measurement benchmarks and requirements. By incorporating health app usability testing throughout the design process, the apps very likely will be useful and usable, and satisfying for users to use.

When developing health apps, a few other considerations need to be made: (1) Legal/Regulatory Status. Legal and regulatory status of health apps is critical for the health app users. Users need to be ensured that the app's security procedures comply with all applicable rules and regulations. Health apps may access protected health information, which must comply with HIPAA privacy and security rules; (2) Safety. This is another critical metric for health apps. The apps should provide information on the proper way of using it. Apps need to make the users aware of possible health dangers related with using the apps for different purposes [33]; and (3) Coordination/Negotiation among Provider, Patient, and Payer. One of the emerging trends in health apps is to match a patient with the right doctor, who might be geographically unavailable to the average patients [34]. The concept of virtual clinic through mobile apps can be implemented by collecting necessary information from the patient, organizing it in the cloud and passing it on to its network of specialists. Payers may also need to be involved in this connection and coordination. Apps can be designed to help people identify the best doctors who could treat their condition in the same geographical area, then set up the office visit appointment for the patients. 


\section{CONCLUSIONS}

This paper discusses some of the major obstacles currently in the way of the adoption of mobile health applications for healthcare providers, patients, and the general health app consumers. There are lots of challenges and questions that need to be addressed pertaining to mobile health applications. When looking from the provider's standpoint, the top two benefits of adopting mobile health are time efficiency and cost efficiency. However, all parties in healthcare are not yet convinced that the technology can help them become successful when they do adopt the mobile technologies. Meeting legal requirements for patient privacy as well as patients' own expectations of privacy, technological limitations still need to be overcome. From the patients' and general health app consumers' standpoint, it appears that the biggest challenge is the lack of guidance and usability evaluation when facing the overwhelming number and huge variety of health apps currently available.

The purpose of this paper is to improve the understanding of the challenges of mobile health apps and strategies with the intention of ensuring appropriate design and implementation of the mobile health apps for healthcare providers, patients, and the general public. The proposed classification scheme and additional usability metrics for health app assessment can be used as guidelines for both implementation now and future research on mobile health apps. Future research can be conducted on a framework for assessing and resolving challenges for appropriate design and use, a usability test on healthcare apps to validate the recommended list of metrics, and studies of user acceptance, which would shed some light on the phenomenon of mobile health apps.

\section{REFERENCES}

1. Beatty, A. L., Fukuoka, Y., \& Whooley, M. A. (2013). Using mobile technology for cardiac rehabilitation: a review and framework for development and evaluation. Journal of the American Heart Association, 2(6), 1-8.

2. Broderick, J., Devine, T., Langhans, E., Lemerise, A. J., Lier, S., \& Harris, L. (2014). Designing health literate mobile apps. Discussion Paper, Institute of Medicine, Washington, DC. http://www.iom.edu/Global/Perspectives/2014/HealthLiterateApps.aspx

3. Cheredar, T. (2012). Want better mobile app curation? Meet Hapoose. VentureBeat News. http://venturebeat.com/2012/10/03/hapoose/

4. Constantino, T. (2013). IMS Health identifies opportunities for mobile healthcare apps to drive patient engagement, enhance delivery of care. IMS Institute for Healthcare Informatics. http://www.imshealth.com/portal/site/imshealth/menuitem.c76283e8bf81e98f53c753c71ad8c22a/?vgnextoid=0 b96ccb4c3402410VgnVCM10000076192ca2RCRD

5. Daley, T. (2013). Health care professionals tapping into mobile devices. http://www.health2con.com/news/2013/05/23/health-care-professionals-tapping-into-mobile-devices/

6. Dillon, A. (2001). User acceptance of information technology. In W. Karwowski (Ed.), Encyclopedia of Human Factors and Ergonomics (pp. 1-10). London, England: Taylor \& Francis.

7. Dudley, B. (2014). Fitness gadgets raise privacy concerns under new health insurance rules. The Seattle Times. http://seattletimes.com/html/businesstechnology/2022868569_briercolumn10xml.html

8. Epocrates (2011). Are healthcare professionals experiencing app overload? Epocrates: An Athenahealth Company (pp. http://www.epocrates.com/appoverload/images/Epocrates_Rx\%20App\%20Overload_Infographic.pdf

9. Epocrates (2013). Epocrates 2013 mobile trends report: maximizing multi-screen engagement among clinicians. $\quad$ Epocrates: An Athenahealth Company (pp. 1-10). http://www.epocrates.com/oldsite/statistics/2013\%20Epocrates\%20Mobile\%20Trends\%20Report_FINAL.pdf

10. FDA (2013). Mobile medical applications: guidance for industry and food and drug administration staff. U.S. Food and Drug Administration (pp. 1-43). http://www.fda.gov/downloads/MedicalDevices/.../UCM263366.pdf

11. Fox, S. (2013). Mobile health in context: how information is woven into our lives. Pew Research Center's Internet \& American Life Project. http://www.pewinternet.org/2013/10/22/mobile-health-in-context/

12. Franko, O. I., \& Tirrell, T. F. (2012). Smartphone app use among medical providers in ACGME training programs. Journal of Medical Systems, 36(5), 3135-3139.

13. Ghose, S., \& Dou, W. (1998). Interactive functions and their impacts on the appeal of Internet presence sites. Journal of Advertising Research, 38(2), 29-43. 
14. Halstead-Nussloch, R., \& Reichgelt, H. (2013). Leveraging HCI in teaching mobile, "anytime and everywhere" IT. In W. D. Armitage, R. S. Friedman, \& K. A. Baker (Eds.), Proceedings of the 14th Annual ACM SIGITE Conference on Information Technology Education (pp. 13-18). Orlando, FL, USA, October 10-12.

15. Harrison, R., Flood, D., \& Duce, D. (2013). Usability of mobile applications: literature review and rationale for a new usability model. Journal of Interaction Science, 1(1), 1-16.

16. Ho, K. (2013). Health-e-apps: a project to encourage effective use of mobile health applications. BC Medical Journal, 55(10), 458-460.

17. Huang, G. T. (2014). Doctors going mobile, but still skeptical of connected health. http://www.xconomy.com/boston/2014/04/08/doctors-going-mobile-but-still-skeptical-of-connected-health/

18. IMS Health (2013). Patient apps for improved healthcare: from novelty to mainstream. IMS Institute for Healthcare Informatics (pp. 1-65). http://www.imshealth.com/deployedfiles/imshealth/Global/Content/Corporate/IMS\%20Health\%20Institute/Rep orts/Patient_Apps/IIHI_Patient_Apps_Report.pdf

19. ISO 9241-11 (1998). Ergonomic requirements for office work with visual display terminals (VDTs) - Part 11: Guidance on usability. International Organization for Standardization. http://www.iso.org/iso/catalogue_detail.htm?csnumber=16883

20. Kalyani, M. (2013). Privacy concerns with mobile health apps. SpiderOak. https://spideroak.com/privacypost/cloud-security/privacy-concerns-with-mobile-health-apps-draft/

21. Kanter, B. (2011). Content curation primer. http://www.bethkanter.org/content-curation-101/

22. Lobo, D., Kaskaloglu, K., Kim, C. Y., \& Herbert, S. (2011). Web usability guidelines for smartphones: a synergic approach. International Journal of Information and Electronics Engineering, 1(1), 33-37.

23. Lowry, S. Z., Quinn, M. T., Ramaiah, M., Schumacher, R. M., Patterson, E. S., North, R., Zhang, J., Gibbons, M. C., \& Abbott, P. (2012). Technical evaluation, testing, and validation of the usability of electronic health records. U.S. Department of Commerce, National Institute of Standards and Technology (NIST): NISTIR 7804.

24. mHIMSS (2012). Selecting a mobile app: evaluating the usability of medical applications. mHIMSS App Usability Work Group (pp. 1-32). http://himss.files.cms-plus.com/HIMSSguidetoappusabilityv1mHIMSS.pdf

25. Mosa, A. S. M., Yoo, I., \& Sheets, L. (2012). A systematic review of healthcare applications for smartphones. BMC Medical Informatics and Decision Making, 12(67), 1-31.

26. O’Neill, S., \& Brady, R. R. (2012). Colorectal smartphone apps: opportunities and risks. Colorectal Disease 14(9), 530-534.

27. research2guidance (2013). Mobile health market report 2013-2017. The Commercialization of mHealth Applications, 3, 1-115.

28. Rubin, J., \& Chisnell, D. (2011). Handbook of usability testing: how to plan, design, and conduct effective tests. Hoboken, NJ: John Wiley \& Sons.

29. Seffah, A., Donyaee, M., Kline, R. B., \& Padda, H. K. (2006). Usability measurement and metrics: a consolidated model. Software Quality Journal, 14(2), 159-178.

30. Sifferlin, A. (2013). Bad news about your favorite health apps: they don't work. Time. http://healthland.time.com/2013/10/31/bad-news-about-your-favorite-health-apps-they-dont-work/

31. Tyer, D. (2013). Mobile health apps - still a novelty? http://www.pmlive.com/blogs/digital_intelligence/archive/2013/november/mobile_health_apps__still_a_novelty

32. van Velsen, L., Beaujean, D. J. M. A., \& van Gemert-Pijnen, J. E. W. C. (2013). Why mobile health app overload drives us crazy, and how to restore the sanity. BMC Medical Informatics \& Decision Making, 13(23), $1-5$.

33. Wenger, G. (2013). EBmH (evidence based mHealth) app development platform. http://prezi.com/tziutsdhsvwv/ebmh-evidence-based-mhealth-app-development-platform/

34. Wicklund, E. (2014). Using mHealth to match a patient with the right doctor. mHealthNews: The Voice of Mobile Healthcare. http://www.mhealthnews.com/news/using-mhealth-match-patient-right-doctor

35. Wierz, D. J., Kurliand, M., \& Christina, J. (2013). Mobile technology revolutionizing healthcare - use case study: the John Hopkins hospital. http://himss.files.cms-plus.com/FileDownloads/2013-mHIMSS-Case-StudyHopkins-Final.pdf

36. World Health Organization (2011). mHealth: new horizons for health through mobile technologies. World Health Organization Global Observatory for eHealth Series, Volume 3. Geneva: WHO Press. http://www.who.int/goe/publications/ehealth_series_vol3/en/ 